\title{
Angle of Attack Sensor for Small Fixed-Wing Unmanned Aerial Vehicles ${ }^{\dagger}$
}

\author{
Kriangkrai Wanngoen ${ }^{1}$, Nanthawat Saetunand ${ }^{1}$, Watcharapol Saengphet ${ }^{2}$ and \\ Suradet Tantrairatn ${ }^{1, *}$ \\ 1 Institute of Engineering, Suranaree University of Technology, Nakhon Ratchasima 30000, Thailand; \\ kriangkrai.w.n@gmail.com (K.W.); nanthawat.jn@gmail.com (N.S.) \\ 2 iCreativeSystem Co. Ltd., Nakhon Ratchasima 30000, Thailand; w.sphet@icsco.ai \\ * Correspondence: suradetj@sut.ac.th; Tel.: +66-955-623-555 \\ + Presented at the Innovation Aviation \& Aerospace Industry-International Conference 2020 (IAAI 2020), \\ Chumphon, Thailand, 13-17 January 2020.
}

Published: 7 January 2020

\begin{abstract}
The angle of attack (AOA) is an important parameter for estimating aerodynamic parameter the performance and stability of aircraft. Currently, AOA sensors are used in general aircraft. However, there is no a reasonable-price AOA sensor that is compatible to a small fixedwing unmanned aerial vehicles (UAVs). This research aims to designs and constructs angle of attract (AOA) sensor for small fixed-wing unmanned aircraft. Mechanism Design, which is similar to aerodynamic wheatear vane, can operate in airspeed $10-30 \mathrm{~m} / \mathrm{s}$. The direction of airfoil aligns with the air flow direction. When the AOA of the UAV changes, the air flow changes the direction, resulting in the change of airfoil direction. The high-resolution rotary encoder, that was used to measure the angle of the airfoil, was installed with the fin airfoil. For experiment, the accuracy of the AOA sensor was validated by comparing the angles obtained from the encoder with the standard rotary table in static and wind tunnel. Finally, the AOA sensor, which was attached on aircraft, was verified and recorded in flight test. As the results of the measurement, the airfoil angles detected by the encoder were in good agreement with the standard angles.
\end{abstract}

Keywords: angle of attack sensor; fixed-wing unmanned aerial vehicles; small unmanned aerial vehicles

\section{Introduction}

The angle of attack (AOA) is an angle between the direction of airflow and the cord-line of the aircraft's wing. It is an essential factor that affects aerodynamic forces. It is also used to determine aircraft performance and stability that help user to know the limit of aircraft. At present, the angle of attack instruments is usually employed for general aircraft to evaluate flight safety. These instruments help to prevent stalling in large and heavy aircraft that usually fly at high speed [1]. However, there is no actual application for using with fixed-wing RC plane, VTOL aircraft, and small power electric fixed-wing UAVs. For the research, the angle of attack instruments is only used for designing the fin.

This paper is aimed to design and create a prototype of the angle of attack instrument that can measure the angle of attack at low speed flight for small fixed-wing UAVs. That can operate at air speed range from 10-30 m/s, using the Sonic Modell Mini Sky hunter V2 EPO which has a flight range of $12-35 \mathrm{~m} / \mathrm{s}$ as a test aircraft. 


\section{Design}

\subsection{Fin Design}

A Fin has asymmetric cross-sectional airfoil. When the symmetric airfoil encounters with airflow, there is a pressure difference between two sides of the fin. This pressure difference induces the fin to move to the lower pressure side. The fin stops moving when it reaches the equilibrium position. At that position, the fin is parallel with the airflow direction. This study designed a mechanism for measuring instrument that is similar to wind vane or weather vane. The fin acted as a rudder while the front arrow acted as a counterweight. The pressure exerted on the fin causes a pitching moment. In the design process, the size of the fin must be suitable to generate the lift force with the small angle of attack at the low speed of airflow. The pitching moment should be higher than the torque fiction of bearing, which can be expressed by Equations (1) and (2).

Lift coefficient [2] and friction moment of bearing [3] can be expressed, respectively

$$
L=\frac{1}{2} \rho v^{2} S C_{l} \quad ; \quad M=0.5 \mu P d
$$

From Momentum equation, that can rewrite in the form of pitching moment as follows

$$
\text { Pitching moment }(P)=\text { Lift } \times \text { Length }(\text { Center of pressure }- \text { Pivot point })
$$

\subsection{Angle Sensor and Electronics Component}

The main factor of choosing an electronic device for the small UAV must be lightweight, durable, and accurate. This study used a high-resolution rotary encoder (As5048a), which is a magnetic position sensor with low friction, to measure the position. This sensor can transmit two types of output data: SPI data and PWM data. The finest resolution that the sensor can measure is 0.06 degrees (in the form of SPI data) [4]. The Arduino Nano board that is small and light was utilized to read the data and convert the data into I2C data before sending it to the Pixhawk4 board (Figure 1). The primary function of the Pixhawk4 board is to control the flight and save flight information.



Figure 1. Pixhawk4 board, Arduino Nano boar, As5048a sensor.

\section{Method}

\subsection{Sensor Assembly}

The data from the datasheet [4] was used to design and build parts that can be installed on small aircraft easily. The 3D elements were made of PLA plastic, and they were created using a 3D printer with a layer thickness of $0.006 \mathrm{~mm}$. All parts were assembled to be angle sensor, as shown in Figure 3. Then, the Arduino Nano was connected to read signal from the As5048a sensor in the form of SPI data (Figure 2). 


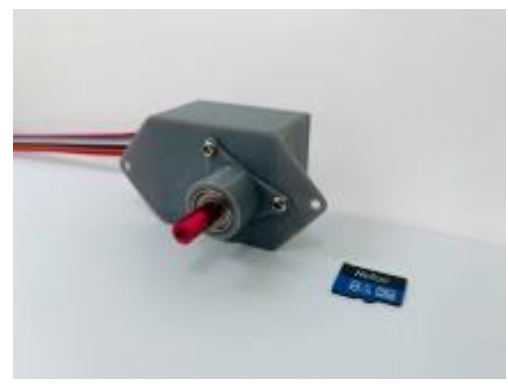

Figure 2. Angle sensor.

\subsection{Angle Sensor and Fin Calibration}

Therefore, the rotary table was chosen to calibrate with a high resolution up to 0.002 degrees [5]. The angle sensor was installed on the rotary table, as in Figure 2. We adjust the rotary angle of 5 degrees per step, then read and record the angle from the Arduino data monitor. The data obtained from the rotary table was compared with the data collected from the angle sensor to compute the smallest error of each step. The angle sensor was performed again by adjusting the rotary from 0.1 degrees to 30 degrees for the finest resolution of the measurement.

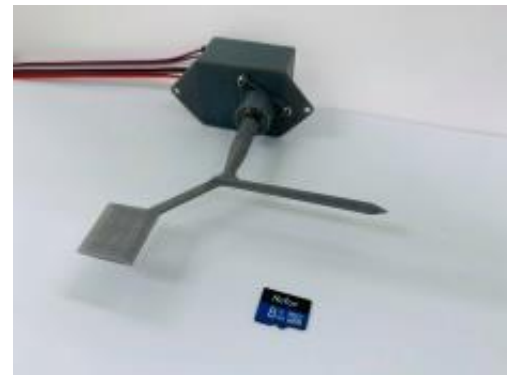

Figure 3. AOA sensor.

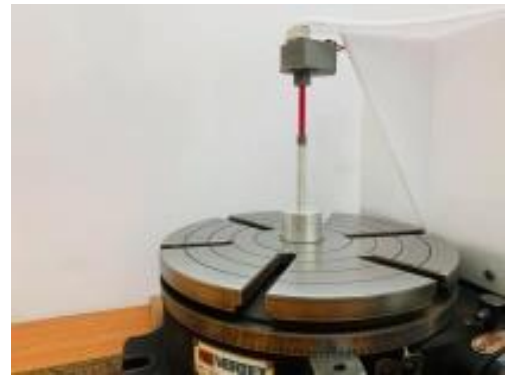

Figure 4. Calibration sensor.

When air flow through the fin, there is a lift force that causes the change in the direction of the fin. The fin always balances itself to be in the equilibrium condition. The fin balancing can be affected by the wind direction, airfoil shape, air speed, fin forming, etc. Therefore, to ensure that the fin is in the position aligned with the direction of the airflow. It is necessary to test the fin in the wind tunnel. This study designed fin that can operate at a minimum speed of $10 \mathrm{~m} / \mathrm{s}$ using NACA 009 airfoil with the cord of $20 \mathrm{~mm}$, and the span of $20 \mathrm{~mm}$. The distance from the pivot point to the center of pressure (Cp) is $25 \mathrm{~mm}$, Reynold number at speed $5 \mathrm{~m} / \mathrm{s}$ is 7000 [6], and $\mathrm{Cl}$ at the angle of attack of 0.1 degrees is 0.006. From Equation (1), we have $L=49.32 \mu \mathrm{N}$. From Equation (2), $\mathrm{P}=1.23 \mu \mathrm{N}$. From Equation (2), $\mathrm{M}=375 \mathrm{Nm}$ that shows that $\mathrm{P}=3.3 \mathrm{M}$. It indicated that the fin could respond to the air speed of $5 \mathrm{~m} / \mathrm{s}$ with the threshold angle of attack of 0.1 degrees. After forming the fin using the 3D printer, the fin was installed into the angle sensor, as shown in Figure 4. The assembly angle sensor was tested in subsonic wind tunnel $305 \mathrm{~mm}$ that was able to generate wind speed of 0-36 m/s. The first experiment was to find the speed range that the fin can work efficiently. The experiment conducted in the range 
of air speed of $0-36 \mathrm{~m} / \mathrm{s}$ with various levels of the angle of attack to test the angle measuring capability of the angle sensor. The correct calibration requires a high precision instrument.

The AOA sensor was installed on the aircraft at the position where the air turbulence was minimum, such as at the front of the plane, etc. The AOA sensor on the aircraft was connected to the flight control board using the data cable. The Arduino Nano board read the data from the magnetic sensor in the form of bit data and then transformed it into I2C data and sends it to the Pixhawk board to receive the data and save the data.

\subsection{Flight Test}

The aircraft equipped with the angle sensor was tested by flying at the height of $100 \mathrm{~m}$. The flight mode was changed to loiter mode with the bank angle of 5 degrees to reduce the influence of side slip turn go. The flight speed was at 18, 21, 24, 27, and $30 \mathrm{~m} / \mathrm{s}$. The test time for each level of wind speed test is one cycle of flight.

\section{Result}

After the re-calibration of the angle sensor, the AOA sensor was tested to find the optimum air speed range that the sensor could function. In the wind tunnel, it was found that the fin stably responded at the air speed range of $10-30 \mathrm{~m} / \mathrm{s}$. It demonstrated that the AOA sensor could work appropriately at the air speed range of $10-30 \mathrm{~m} / \mathrm{s}$. When the angle sensor is rotated by 5 degrees at the air speed range of $10-30 \mathrm{~m} / \mathrm{s}$ to measure the angle of the fin and the angle sensor, it is found that the measured angle of the fin and the measured angle collected from the angle sensor had an average difference of 5 degrees. It demonstrated that the responding angle of the fin was in good agreement with the measured angle collected from the angle sensor, as shown in Figure 5. Therefore, this AOA sensor can work properly during Air velocity from $10-30 \mathrm{~m} / \mathrm{s}$.

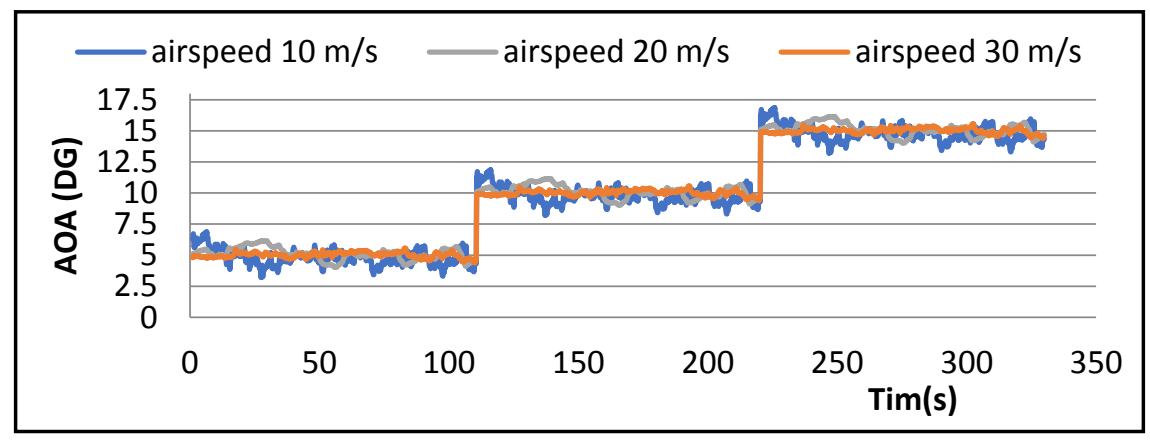

Figure 5. Calibration Angle of fin-Angle of sensor.

\section{Flight Test}

Figure 6 shows the relationship between the speed of aircraft and the angle of attack measured in each unit of time. Test speeds start from $18-30 \mathrm{~m} / \mathrm{s}$. Because the batteries of test aircraft were increased during the test, increasing the load, and the flight speed to avoid the stalling. In this test, it could fly at a minimum speed of $18 \mathrm{~m} / \mathrm{s}$. From the above information, when an aircraft flew at the lowest speed of $18 \mathrm{~m} / \mathrm{s}$, the angle of attack was in between the range of 12-13 degrees. While at a maximum speed of $30 \mathrm{~m} / \mathrm{s}$, the angle of attack was in between the range of 1-1.5 degrees. When the aircraft operated at a constant level of height with the speed reduction, the aircraft needed to increase the angle of attack to create enough lift force to fly at the same altitude. On the other hand, if the aircraft increased in speed, it must reduce the angle of attack to maintain itself at the same height. In this experiment, the flying height was at $100 \mathrm{~m}$ for all test speeds. From Figure 7, it demonstrates that the AOA sensor can operate on small aircraft at a flight speed of $10-30 \mathrm{~m} / \mathrm{s}$. 


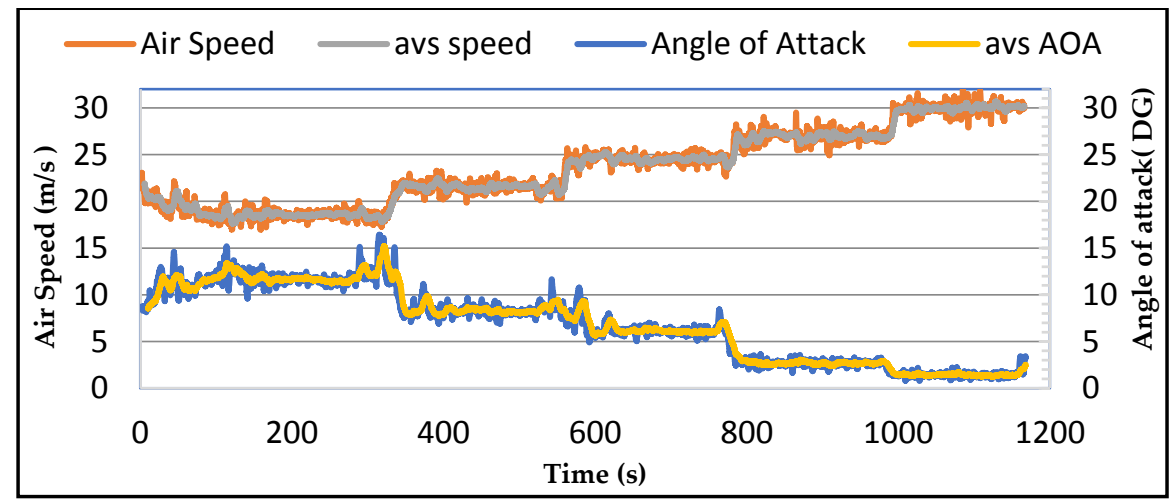

Figure 7. Relation of airspeed and AOA in actual flight.

\section{Conclusions}

The AOA sensor for small aircraft has two main components: a mechanism for measuring wind direction and a sensor for measuring the angle of rotation of the device. In mechanical design, it must have an asymmetric cross-sectional shape to be able to respond to the change in the wind direction as low as 1 degree. The AOA sensor can work at air speed that is lower or equal to $10 \mathrm{~m} / \mathrm{s}$. The sensor selection must be small, light, and high accuracy.

This study designed and built the AOA sensor that could measure the angle of attack at the air speed of $10-30 \mathrm{~m} / \mathrm{s}$ with a weather vane-like mechanism. The fin was a NACA 009 that could respond to the air speed of 10-30 m/s stably. There was a good agreement between the measured angle of the fin and the measured angle collected from the angle sensor. Therefore, this AOA sensor can work properly during Air velocity from $10-30 \mathrm{~m} / \mathrm{s}$.

\section{References}

1 Angle of Attack (AOA) Systems. Available online: https://utcaerospacesystems.com/wpcontent/uploads/2018/04/Angle-of-Attack-AOA-Systems.pdf (accessed on 10 November 2019).

2 Anderson, J.D., Jr. Introduction to Flight, 3rd ed.; McGraw-Hill Education: New York, NY, USA, 1976; p. 184.

3 General Catalogue; Svenska Kullagerfabriken: Media-Print, Germany, 2003; p. 88.

4 AS5048A High-Resolution Position Sensor. Available online: https://ams.com/as5048a (accessed on 8 October 2019).

5 Prieto, E.; Pérez, M.D.; Yandayan, T.; Przybylska, J.; Just, A.; Geckeler, R. General Catalogue; EURAMET e.V.: Braunschweig, Germany, 2018; p. 12.

6 Reynolds Number Calculation. Available online: http://airfoiltools.com/calculator/reynoldsnumber accessed on 8 October 2019).

(C) 2020 by the authors. Licensee MDPI, Basel, Switzerland. This article is an open access article distributed under the terms and conditions of the Creative Commons Attribution (CC BY) license (http://creativecommons.org/licenses/by/4.0/). 